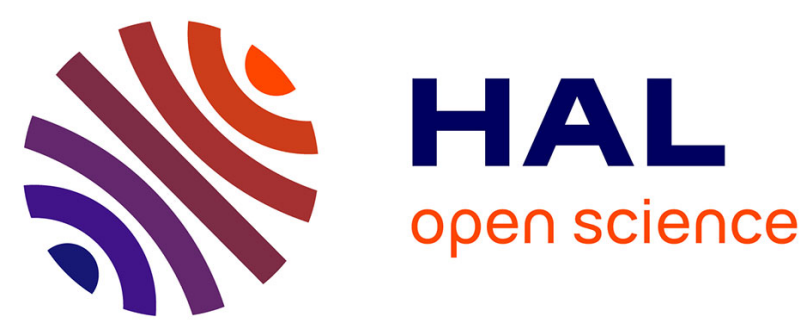

\title{
Full-Duplex versus Half-Duplex Large Scale Antenna System
}

\author{
Arman Shojaeifard, Kai-Kit Wong, Marco Di Renzo, Gan Zheng, Khairi
}

Ashour Hamdi, Jie Tang

\section{- To cite this version:}

Arman Shojaeifard, Kai-Kit Wong, Marco Di Renzo, Gan Zheng, Khairi Ashour Hamdi, et al.. FullDuplex versus Half-Duplex Large Scale Antenna System. 2017 IEEE International Conference on Communications Workshops (ICC Workshops), May 2017, Paris, France. 10.1109/ICCW.2017.7962747. hal-01880003

\section{HAL Id: hal-01880003 \\ https://hal.science/hal-01880003}

Submitted on 15 Jul 2020

HAL is a multi-disciplinary open access archive for the deposit and dissemination of scientific research documents, whether they are published or not. The documents may come from teaching and research institutions in France or abroad, or from public or private research centers.
L'archive ouverte pluridisciplinaire HAL, est destinée au dépôt et à la diffusion de documents scientifiques de niveau recherche, publiés ou non, émanant des établissements d'enseignement et de recherche français ou étrangers, des laboratoires publics ou privés. 


\title{
Full-Duplex versus Half-Duplex Large Scale Antenna System
}

\author{
Arman Shojaeifard*, Kai-Kit Wong*, Marco Di Renzo ${ }^{\dagger}$, Gan Zheng ${ }^{\ddagger}$, Khairi Ashour Hamdi ${ }^{\S}$, Jie Tang $₫$ \\ *Communications and Information Systems Group, University College London, London, United Kingdom \\ ${ }^{\dagger}$ Laboratoire des Signaux et Systèmes, CNRS, CentraleSupélec, Univ Paris Sud, Université Paris-Saclay, Paris, France \\ ${ }_{\ddagger}^{\ddagger}$ Signal Processing and Networks Research Group, Loughborough University, Loughborough, United Kingdom \\ $\S$ Microwave and Communication Systems Group, University of Manchester, Manchester, United Kingdom \\ TSmart Information Processing Centre, South China University of Technology, Guangzhou, China \\ E-Mail: *a.shojaeifard@ucl.ac.uk; *kai-kit.wong@ucl.ac.uk; ${ }^{\dagger}$ marco.direnzo@12s.centralesupelec.fr; \\ †g.zheng@lboro.ac.uk; §k.hamdi@manchester.ac.uk; 『eejtang@scut.edu.cn
}

\begin{abstract}
In this paper, we provide a unified framework for the study of multi-cell multi-user large-scale antenna system (LSAS) in both full-duplex (FD) and half-duplex (HD) modes of communications. Here, we employ the Poisson point process (PPP)-based abstraction model of massive-antenna base stations (BSs) and mobile terminals (MTs). The loop-back interference (LI) channels in FD mode are modeled using the Rician distribution. All other channels are characterized using the Rayleigh distribution, including the cross downlink (DL)-uplink (UL) interference $(\mathrm{CI})$ in FD mode. The signals statistical distributions under different linear conjugate-beamforming (CF) and zero-forcing $(\mathrm{ZF})$ processes are derived. We develop analytical expressions for computing the LSAS DL and UL ergodic rates (ERs) and further study the performance gain of FD versus HD. The results highlight that the corresponding FD over HD ER gain increases only logarithmically in the antenna array size.
\end{abstract}

\section{INTRODUCTION}

Large scale antenna system (LSAS), or massive multipleinput multiple-output (MIMO), is considered a key enabler for future fifth-generation $(5 \mathrm{G})$ cellular network. The LSAS structure comprises base stations (BSs), each equipped with massive-antennas, capable of serving many mobile terminals (MTs) over the same temporal/spectral bins. The high antenna array gain in LSAS facilitates achieving radical performance improvements versus the existing wireless standards. SoftBank, one of the first operators in the world to deploy massive MIMO technology (using 128-antenna arrays across 100 macro-cells), has recently reported up to a ten-fold increase in capacity [1].

LSAS, in the context of half-duplex (HD) systems, where the downlink (DL) and uplink (UL) communication directions are separated in time or frequency, has been rigorously investigated in the past, see, e.g., [2], [3] and the references therein. In full-duplex (FD) mode, the transmit and receive functionalities occur simultaneously over the same radio resources, resulting in increased interference level and complexity [4][6]. Recently, LSAS, has gained a lot of attention as a candidate solution for unlocking the full potential of large scale FD deployment through improved resilience against loop-back interference (LI) and cross DL-UL interference (CI).
In [7], the ergodic rates (ERs) between two FD nodes with massive number of antennas were studied under different LI suppression and subtraction schemes. The authors in [8] compared the FD versus HD ERs in a single-cell LSAS by utilizing the excessive transmit antennas to (spatially) suppress LI at the receiver side. Multi-cell studies of LSAS with CI have also been recently reported. In [9], the DL and UL ERs in a deterministic FD multi-cell multi-user MIMO setup were characterized. The anticipated two-fold ER gain of FD compared to HD was shown to remain in the asymptotic antenna region [9]. On the other hand, random FD cellular networks with directional antennas were studied in [10]. In particular, the authors provided analytical expressions for the DL and UL coverage probabilities using stochastic geometry theory.

Here, we provide a generalized framework for the performance analysis of FD and HD multi-cell multi-user LSAS. This work differs from [9] in that the nodes are drawn from the Poisson point process (PPP)-based abstraction model. This approach complicates the analysis but is considered more useful for depicting design insights [11], for instance, via capturing large-scale fading, cellular association, and dense topologies. In relation to [10], the array gains are not assumed to be fixed linear functions in the number of antennas and streams here; rather they are treated as random variables having unique statistical properties. Furthermore, in this work, we study the more involved ER performance, with the average being taken over both the spatial process and channel propagation.

The framework presented is in some sense an extension of [12] as we consider both linear conjugate-beamforming (CB) and zero-forcing (ZF) processes, as well as both LI suppression and subtraction schemes. We characterize the statistical distributions of all signals in the multi-cell multiuser LSAS, including, in the case of FD mode, a novel tight approximation of the LI at the massive-antenna BSs over Rician fading channels. Moreover, tractable analytical expressions for the computation of the LSAS ERs in the DL and UL are provided. In addition, the performance gain of FD versus HD LSAS is investigated using theoretical and simulation results. 
Notation: $\mathbf{X}$ is a matrix with $(n, m)$-th element $\{\mathbf{X}\}_{n, m}$; $\mathbf{x}$ is a vector with $k$-th entry $\{\mathbf{x}\}_{k} ; \mathrm{T}$ is the transpose; $\dagger$ is the conjugate-transpose; + is the pseudo-inverse; $|$.$| is the$ modulus; $\|$.$\| is the norm; \mathbb{1}($.$) is the indicator function; \mathcal{E}\{$. is the average; $\mathcal{V}\{$.$\} is the variance; \mathcal{M}_{(.)}(z)$ is the moment generating function (MGF); $\mathcal{P}($.$) is the probability density$ function (PDF); $\Gamma($.$) is the Gamma function; \Gamma(.,$.$) is the$ incomplete Gamma function; $\mathcal{B}_{z}(.,$.$) is the incomplete beta$ function; $\mathcal{C N}\left(\mu, \nu^{2}\right)$ is the complex Gaussian distribution with mean $\mu$ and variance $\nu^{2}$; and $\mathcal{G}(\kappa, \theta)$ is the Gamma distribution with shape and scale parameters $\kappa$ and $\theta$, respectively.

\section{LSAS MODELING}

Consider a multi-cell multi-user LSAS setup with BSs and MTs respectively deployed on the Euclidean grid according to independent stationary PPPs $\Phi_{\mathrm{BS}}$ and $\Phi_{\mathrm{T}}$ with spatial densities $\lambda_{\mathrm{BS}}$ and $\lambda_{\mathrm{T}}$ [13]. The transmit and receive antennas at the BS side are denoted with $M$ and $N$, respectively. Each deployed massive-antenna BS is assumed to communicate with respect to $K(\leq \min (M, N))$ active MTs. Each MT, in compliance with the increasing restriction on the physical dimensions of the devices, is equipped with two antennas; one for transmission, and the other for reception [14]. Let $p_{\mathrm{BS}}$ and $p_{\mathrm{MT}}$ respectively represent the transmit powers at the massiveantenna BSs (per user) and MTs. We consider both FD and HD modes of communications here. In FD mode, the DL and UL functions take place over the same time/frequency resources, giving rise to new CI and LI terms [15]. In HD mode, on the other hand, the different DL and UL communications directions are separated orthogonally in the time-domain.

In this paper, we employ a cellular association strategy, where each MT exclusively communicates in both DL and UL with a massive-antenna BS which provides the greatest received signal power. Let $\Phi_{\mathrm{MT}}\left(\subset \Phi_{\mathrm{T}}\right)$ denote the set of active MTs. We denote the location of the $j$-th massive-antenna BS with $j_{0}\left(\in \Phi_{\mathrm{BS}}\right)$, and its $k$-th $(\in \mathcal{K}=\{1, \cdots, K\})$ active MT with $j_{k}\left(\in \Phi_{\mathrm{MT}}\right)$, respectively. By invoking the SlivnyakMecke theorem, (i) in the DL, the analysis is computed for a typical active MT $l_{k}\left(\in \Phi_{\mathrm{MT}}\right)$ assumed to be at the origin, and (ii) in the UL, the analysis is realized for the transmitted signal from $l_{k}$ at its serving massive-antenna BS $l_{0}\left(\in \Phi_{\mathrm{BS}}\right)$. By employing the distance-dependent pathloss model with exponent $\beta(>2)$, the cellular association strategy is $l_{0}=\arg \min \left(d_{\left[j_{0}, l_{k}\right]}^{\beta}\right)$ where $d_{\left[j_{0}, l_{k}\right]}=\left\|j_{0}-l_{k}\right\|$. The corresponding transceiver distance PDF is given by $\mathcal{P}_{d_{\left[l_{0}, l_{k}\right]}}(r)=2 \pi \lambda_{\mathrm{BS}} r \exp \left(-\pi \lambda_{\mathrm{BS}} r^{2}\right)[16]$.

Let $\mathbf{g}_{\left[j_{k}, j_{0}\right]} \in \mathcal{C}^{1 \times M}$ and $\mathbf{g}_{\left[j_{0}, j_{k}\right]} \in \mathcal{C}^{N \times 1}$ denote the DL and UL channels between the massive-antenna BS $j_{0}$ and active MT $j_{k}$, respectively. The combined DL and UL channels are respectively given by $\mathbf{G}_{\left[j_{K}, j_{0}\right]}=\left[\mathbf{g}_{\left[j_{k}, j_{0}\right]}^{\boldsymbol{\top}}\right]_{1 \leq k \leq K}^{\top} \in \mathcal{C}^{K \times M}$ and $\mathbf{G}_{\left[j_{0}, j_{K}\right]}=\left[\mathbf{g}_{\left[j_{0}, j_{k}\right]}\right]_{1 \leq k \leq K} \in \mathcal{C}^{N \times K}$. We denote the $\mathrm{CI}$ channels at the massive-antenna BS $l_{0}$ from the massiveantenna BS $j_{0}$ with $\mathbf{G}_{\left[l_{0}, j_{0}\right]} \in \mathcal{C}^{N \times M}$, and at the active MT $l_{k}$ from the active MT $j_{k}$ with $g_{\left[l_{k}, j_{k}\right]}$, respectively. Moreover, the LI channels at the massive-antenna BS $j_{0}$ and active MT $j_{k}$ are respectively represented using $\mathbf{G}_{\left[j_{0}, j_{0}\right]} \in \mathcal{C}^{N \times M}$ and $h_{\left[j_{k}, j_{k}\right]}$. The LI channels are subject to Rician fading with independent elements distributed according to $\mathcal{C N}\left(\mu, \nu^{2}\right)$. The other channels are subject to Rayleigh fading with independent elements generated from $\mathcal{C N}(0,1)$. We consider channel state information (CSI) to be available through UL pilot training [3].

Let $\eta_{\left[l_{k}, l_{0}\right]}$ denote the DL complex additive white Gaussian noise (AWGN) with mean zero and variance $\sigma_{\mathrm{DL}}^{2}$ at the reference active MT $l_{k}$. Moreover, we use $\eta_{\left[l_{0}, l_{k}\right]} \in \mathcal{C}^{N \times 1}$ to represent the UL circularly-symmetric complex AWGN vector with mean zero and covariance matrix $\sigma_{\mathrm{UL}}^{2} \mathbf{I}_{N}$ at the reference massive-antenna BS $l_{0}$.

\section{LINEAR PROCESSING}

We can describe the arbitrary linear precoding/decoding design at the multi-antenna BSs as follows. Let $\mathbf{s}_{\left[j_{K}, j_{0}\right]}=$ $\left[s_{\left[j_{k}, j_{0}\right]}\right]_{1 \leq k \leq K}^{\top} \in \mathcal{C}^{K \times 1}, \mathcal{E}\left\{\left|s_{\left[j_{k}, j_{0}\right]}\right|^{2}\right\}=1$, denote the DL complex information vector from the massive-antenna BS $j_{0}$ with respect to all its active MTs $j_{k}, k \in \mathcal{K}$. In the reverse direction of communications, the UL complex information vector at the massive-antenna BS $j_{0}$ from all its active MTs $j_{k}, k \in \mathcal{K}$, is $\mathbf{s}_{\left[j_{0}, l_{K}\right]}=\left[s_{\left[l_{0}, l_{k}\right]}\right]_{1 \leq k \leq K}^{\top} \in$ $\mathcal{C}^{K \times 1}, \mathcal{E}\left\{\left|s_{\left[l_{0}, l_{k}\right]}\right|^{2}\right\}=1$. The transmit signal vector with linear precoding at the massive-antenna BS $j_{0}$ is hence given by $\mathbf{x}_{\left[j_{K}, j_{0}\right]}=\mathbf{V}_{\left[j_{K}, j_{0}\right]} \mathbf{S}_{\left[j_{K}, j_{0}\right]}$ where $\mathbf{V}_{\left[j_{K}, j_{0}\right]}=$ $\left[\mathbf{v}_{\left[j_{k}, j_{0}\right]}\right]_{1 \leq k \leq K} \in \mathcal{C}^{M \times K}$ is the corresponding precoding matrix. In addition, the linear receive filter at the massive-antenna BS $j_{0}$ is defined as $\mathbf{W}_{\left[j_{0}, j_{K}\right]}=\left[\mathbf{w}_{\left[j_{0}, j_{k}\right]}^{\top}\right]_{1 \leq k \leq K}^{\top} \in \mathcal{C}^{K \times N}$.

Next, we derive generalized expressions for the received signal-to-interference-plus-noise ratios (SINRs) under arbitrary FD/HD mode and linear precoder/decoder design.

In the DL, the SINR at the reference active MT in the LSAS paradigm is given by

$\mathcal{Y}_{\mathrm{DL}}=\frac{\mathcal{X}_{\mathrm{DL}}}{\mathcal{M} \mathcal{U} \mathcal{I}_{\mathrm{DL}}+\mathcal{I C I}_{\mathrm{DL}}+\mathbb{1}_{\mathrm{FD}}\left(\mathcal{C} \mathcal{I}_{\mathrm{DL}}\right)+\mathbb{1}_{\mathrm{FD}}\left(\mathcal{L I}_{\mathrm{DL}}\right)+\mathcal{N}_{\mathrm{DL}}}$

where $\mathcal{X}_{\mathrm{DL}}=p_{\mathrm{BS}} d_{\left[l_{k}, l_{0}\right]}^{-\beta} G_{\mathcal{X}_{\mathrm{DL}}}, G_{\mathcal{X}_{\mathrm{DL}}}=\left|\mathbf{g}_{\left[l_{k}, l_{0}\right]} \mathbf{v}_{\left[l_{k}, l_{0}\right]}\right|^{2}$, $\mathcal{M U \mathcal { I } _ { \mathrm { DL } }}=p_{\mathrm{BS}} d_{\left[l_{k}, l_{0}\right]}^{-\beta} G_{\mathcal{M U} \mathcal{I}_{\mathrm{DL}}}, G_{\mathcal{M U} \mathcal{I}_{\mathrm{DL}}}=\sum_{u \in \mathcal{K} \backslash\{k\}}$ $\left|\mathbf{g}_{\left[l_{k}, l_{0}\right]} \mathbf{v}_{\left[l_{u}, l_{0}\right]}\right|^{2}, \mathcal{I C}_{\mathcal{I}_{\mathrm{DL}}}=p_{\mathrm{BS}} \sum_{j_{0} \in \Phi_{\mathrm{BS}} \backslash\left\{l_{0}\right\}} d_{\left[l_{k}, j_{0}\right]}^{-\beta} G_{\mathcal{I C} \mathcal{I}_{\mathrm{DL}}}$, $G_{\mathcal{I C I}_{\mathrm{DL}}}=\left\|\mathbf{g}_{\left[l_{k}, j_{0}\right]} \mathbf{V}_{\left[j_{K}, j_{0}\right]}\right\|^{2}, \mathcal{C} \mathcal{I}_{\mathrm{DL}}=p_{\mathrm{MT}} \sum_{j_{k} \in \Phi_{\mathrm{MT}} \backslash\left\{l_{k}\right\}}$ $d_{\left[l_{k}, j_{k}\right]}^{-\beta} G_{\mathcal{C I}_{\mathrm{DL}}}, G_{\mathcal{C I}_{\mathrm{DL}}}=\left|g_{\left[l_{k}, j_{k}\right]}\right|^{2}, \quad \mathcal{L I}_{\mathrm{DL}}=p_{\mathrm{MT}} G_{\mathcal{L I}_{\mathrm{DL}}}$, $G_{\mathcal{L I}_{\mathrm{DL}}}=\left|g_{\left[l_{k}, l_{k}\right]}\right|^{2}$, and $\mathcal{N}_{\mathrm{DL}}=\left|\eta_{\mathrm{DL}}\right|^{2}$.

In addition, in the UL of the LSAS, the post-processing SINR at the reference massive-antenna BS is given by

$\mathcal{Y}_{\mathrm{UL}}=\frac{\mathcal{X}_{\mathrm{UL}}}{\mathcal{M U I}_{\mathrm{UL}}+\mathcal{I C I}_{\mathrm{UL}}+\mathbb{1}_{\mathrm{FD}}\left(\mathcal{C I}_{\mathrm{UL}}\right)+\mathbb{1}_{\mathrm{FD}}\left(\mathcal{L I}_{\mathrm{UL}}\right)+\mathcal{N}_{\mathrm{UL}}}$

where $\mathcal{X}_{\mathrm{UL}}=p_{\mathrm{MT}} d_{\left[l_{0}, l_{k}\right]}^{-\beta} G_{\mathcal{X}_{\mathrm{UL}}}, G_{\mathcal{X}_{\mathrm{UL}}}=\left|\mathbf{w}_{\left[l_{0}, l_{k}\right]}^{\top} \mathbf{g}_{\left[l_{0}, l_{k}\right]}\right|^{2}$, $\mathcal{M U} \mathcal{I}_{\mathrm{UL}}=p_{\mathrm{MT}} \sum_{u \in \mathcal{K} \backslash\{k\}} d_{\left[l_{0}, l_{u}\right]}^{-\beta} G_{\mathcal{M U}} \mathcal{I}_{\mathrm{UL}}, G_{\mathcal{M U} \mathcal{I}_{\mathrm{UL}}}=\mid \mathbf{w}_{\left[l_{0}, l_{k}\right]}^{\top}$ $\left.\mathbf{g}_{\left[l_{0}, l_{u}\right]}\right|^{2}, \mathcal{I C}_{\mathrm{UL}}=p_{\mathrm{MT}} \sum_{j_{k} \in \Phi_{\mathrm{MT}} \backslash\left\{\forall l_{u}\right\}, u \in \mathcal{K}} d_{\left[l_{0}, j_{k}\right]}^{-\beta} G_{\mathcal{I C} \mathcal{I}_{\mathrm{UL}}}$, $G_{\mathcal{I C} \mathcal{I}_{\mathrm{UL}}}=\left|\mathbf{w}_{\left[l_{0}, l_{k}\right]}^{\top} \mathbf{g}_{\left[l_{0}, j_{k}\right]}\right|^{2}, \mathcal{C} \mathcal{I}_{\mathrm{UL}}=p_{\mathrm{BS}} \sum_{j_{0} \in \Phi_{\mathrm{BS}} \backslash\left\{l_{0}\right\}} d_{\left[l_{0}, j_{0}\right]}^{-\beta}$ $G_{\mathcal{C I}_{\mathrm{UL}}}, G_{\mathcal{C I}_{\mathrm{UL}}}=\left\|\mathbf{w}_{\left[l_{0}, l_{k}\right]}^{\top} \mathbf{G}_{\left[l_{0}, j_{0}\right]} \mathbf{V}_{\left[j_{K}, j_{0}\right]}\right\|^{2}, \mathcal{L} \mathcal{I}_{\mathrm{UL}}=p_{\mathrm{BS}} G_{\mathcal{L I}_{\mathrm{UL}}}$ $, G_{\mathcal{L I}_{\mathrm{UL}}}=\left\|\mathbf{w}_{\left[l_{0}, l_{k}\right]}^{\top} \mathbf{G}_{\left[l_{0}, l_{0}\right]} \mathbf{V}_{\left[l_{K}, l_{0}\right]}\right\|^{2}, \mathcal{N}_{\mathrm{UL}}=\left|\mathbf{w}_{\left[l_{0}, l_{k}\right]}^{\top} \eta_{\mathrm{UL}}\right|^{2}$. 


\begin{tabular}{|c|c|c|c|c|c|c|c|c|}
\hline Precoder $(\mathbf{V}) /$ Decoder $(\mathbf{W})$ & $G_{\mathcal{X}_{\mathrm{DL}}}$ & $G_{\mathcal{X}_{\mathrm{UL}}}$ & $G_{\mathcal{M U I} \mathcal{I}_{\mathrm{DL}}}$ & $G_{\mathcal{M U} \mathcal{I}_{\mathrm{UL}}}$ & $G_{\mathcal{I C I} \mathcal{I L}_{\mathrm{DL}}}$ & $G_{\mathcal{I C I}_{\mathrm{UL}}}$ & $G_{\mathcal{C} \mathcal{I}_{\mathrm{DL}}}$ & $G_{\mathcal{C I} \mathcal{I}_{\mathrm{UL}}}$ \\
\hline $\mathrm{CB} / \mathrm{CB}$ & $\kappa=M$ & $\kappa=N$ & $\kappa=K-1$ & $\kappa=1$ & $\kappa=K$ & $\kappa=1$ & $\kappa=1$ & $\kappa=K$ \\
\hline CB-N / CB & $\kappa=M-N$ & $-"-$ & $-"-$ & -"- & $-"-$ & $-"-$ & $-"-$ & $-"-$ \\
\hline $\mathrm{ZF} / \mathrm{CB}$ & $\kappa=M-K+1$ & $-"-$ & 0 & $-"-$ & -"- & -"- & -"- & $-"-$ \\
\hline ZF-N / CB & $\kappa=M-N-K+1$ & $-"-$ & $-"-$ & $-"-$ & $-"-$ & $-"-$ & $-"-$ & $-"-$ \\
\hline $\mathrm{CB} / \mathrm{ZF}$ & $\kappa=M$ & $\kappa=N-K+1$ & $\kappa=K-1$ & 0 & -"- & -"- & -"- & $-"$ \\
\hline $\mathrm{CB}-\mathrm{N} / \mathrm{ZF}$ & $\kappa=M-N$ & $-"-$ & -"- & $-"-$ & -"- & -"- & -"- & $-"-$ \\
\hline $\mathrm{ZF} / \mathrm{ZF}$ & $\kappa=M-K+1$ & $-"-$ & 0 & -"- & -"- & $-"-$ & $-"-$ & $-"-$ \\
\hline ZF-N / ZF & $\kappa=M-N-K+1$ & -"- & -"- & -"'- & -"'- & -"- & $-"-$ & $-"-$ \\
\hline
\end{tabular}

TABLE I: DL and UL signals (excluding LI) in the multi-cell multi-user LSAS distributed according to $\mathcal{G}(\kappa, 1)$.

\begin{tabular}{|c|c|c|}
\hline Precoder (V) & $G_{\mathcal{L I}}$ & $G_{\mathcal{L}}$ \\
\hline CB & $\kappa=\frac{\left(\mu^{2}+\nu^{2}\right)^{2}}{\left(2 \mu^{2}+\nu^{2}\right) \nu^{2}}, \theta=\frac{\left(2 \mu^{2}+\nu^{2}\right) \nu^{2}}{\mu^{2}+\nu^{2}}$ & $\kappa=\frac{K\left(\mu^{2}+\nu^{2}\right)^{2}}{(K+2) \mu^{4}+2 \mu^{2} \nu^{2}+\nu^{4}}, \theta=\frac{(K+2) \mu^{4}+2 \mu^{2} \nu^{2}+\nu^{4}}{\mu^{2}+\nu^{2}}$ \\
\hline ZF & $-"-$ & $-{ }^{2}-$ \\
\hline CB-N & $-"-$ & 0 \\
\hline ZF-N & $-"-$ & - \\
\hline
\end{tabular}

TABLE II: DL and UL LI signals in the multi-cell multi-user LSAS distributed according to $\mathcal{G}(\kappa, \theta)$.

In the DL, the reference massive-antenna BS $l_{0}$ applies any of the following linear precoders:

$$
\mathrm{CB}: \mathbf{V}_{\left[l_{K}, l_{0}\right]}=\mathbf{G}_{\left[l_{K}, l_{0}\right]}^{\dagger}
$$

and

$$
\text { ZF: } \mathbf{V}_{\left[l_{K}, l_{0}\right]}=\mathbf{G}_{\left[l_{K}, l_{0}\right]}^{+}=\mathbf{G}_{\left[l_{K}, l_{0}\right]}^{\dagger}\left(\mathbf{G}_{\left[l_{K}, l_{0}\right]} \mathbf{G}_{\left[l_{K}, l_{0}\right]}^{\dagger}\right)^{-1} \text {. }
$$

In FD mode, the transmit antenna array can be utilized for LI suppression using linear CB with LI nulling (CB-N) (conditioned on $M \geq N$ ), or linear $\mathrm{ZF}$ with LI nulling (ZF$\mathrm{N})$ precoders (conditioned on $M \geq N+K$ ):

$$
\begin{gathered}
\text { CB-N: } \mathbf{V}_{\left[l_{K}, l_{0}\right]}=\hat{\mathbf{G}}_{\left[l_{K}, l_{0}\right]}^{\dagger}, \\
\hat{\mathbf{G}}_{\left[l_{K}, l_{0}\right]}=\mathbf{G}_{\left[l_{K}, l_{0}\right]}\left(\mathbf{I}_{M}-\mathbf{G}_{\left[l_{0}, l_{0}\right]}^{\dagger}\left(\mathbf{G}_{\left[l_{0}, l_{0}\right]} \mathbf{G}_{\left[l_{0}, l_{0}\right]}^{\dagger}\right)^{-1} \mathbf{G}_{\left[l_{0}, l_{0}\right]}\right)
\end{gathered}
$$

and

$$
\begin{gathered}
\text { ZF-N: } \mathbf{V}_{\left[l_{K}, l_{0}\right]}=\hat{\mathbf{G}}_{\left[l_{K}, l_{0}\right]}^{+}=\hat{\mathbf{G}}_{\left[l_{K}, l_{0}\right]}^{\dagger}\left(\hat{\mathbf{G}}_{\left[l_{K}, l_{0}\right]} \hat{\mathbf{G}}_{\left[l_{K}, l_{0}\right]}^{\dagger}\right)^{-1}, \\
\hat{\mathbf{G}}_{\left[l_{K}, l_{0}\right]}=\mathbf{G}_{\left[l_{K}, l_{0}\right]}\left(\mathbf{I}_{M}-\mathbf{G}_{\left[l_{0}, l_{0}\right]}^{\dagger}\left(\mathbf{G}_{\left[l_{0}, l_{0}\right]} \mathbf{G}_{\left[l_{0}, l_{0}\right]}^{\dagger}\right)^{-1} \mathbf{G}_{\left[l_{0}, l_{0}\right]}\right) .
\end{gathered}
$$

In the UL, the reference massive MIMO BS $l_{0}$ utilizes any of the following linear decoders:

$$
\mathrm{CB}: \mathbf{W}_{\left[l_{0}, l_{k}\right]}=\mathbf{G}_{\left[l_{0}, l_{K}\right]}^{\dagger}
$$

and

$$
\mathrm{ZF}: \mathbf{W}_{\left[l_{0}, l_{k}\right]}=\left(\mathbf{G}_{\left[l_{0}, l_{K}\right]}^{\dagger} \mathbf{G}_{\left[l_{0}, l_{K}\right]}\right)^{-1} \mathbf{G}_{\left[l_{0}, l_{K}\right]}^{\dagger} .
$$

Lemma 1. Considering linear beamforming over Rayleigh fading channels, we can characterize the statistical distributions of the useful and interfering signals using the Gamma distribution. Hence, with appropriate beam normalization, and assuming that the outer-cell precoding matrices have independent column vectors, we arrive at the different $D L$ and UL channel power gains following $\mathcal{G}(\kappa, 1)$ in Table I.

Proof: The results with linear ZF and ZF-N processes can be found in [12]. The results with linear $\mathrm{CB}$ and $\mathrm{CB}-\mathrm{N}$ beamformers can be similarly obtained.

On the other hand, characterizing the LI over Rician fading channels is challenging, particularly at the massive-antenna BSs (UL). Here, we develop novel tight Gamma approximations using the central limit theorem (CLT) for the different linear processes described previously.

Lemma 2. Considering linear beamforming over Rician fading channels, we can tightly approximate the statistical distributions of the LI signals using the Gamma distribution. With appropriate beam normalization, we arrive at the different $L I$ channel power gains in the DL and $U L$ following $\mathcal{G}(\kappa, \theta)$ as shown in Table II.

Proof: Due to space limitations, we provide only a sketch of the proof. Here, we apply the CLT theorem by deriving the mean and variance of the LI channels. In particular, using tools from random matrix theory, the second and fourth moments of the massive-antenna BS LI channel gain, $\left\|\mathbf{w}_{\left[l_{0}, l_{k}\right]}^{\boldsymbol{\top}} \mathbf{G}_{\left[l_{0}, l_{0}\right]} \mathbf{V}_{\left[l_{K}, l_{0}\right]}\right\|$, in the asymptotic antenna region, $M \rightarrow \infty, N \rightarrow \infty$, can be derived as $K\left(\mu^{2}+\nu^{2}\right)$ and $K(K+1)\left(2 \mu^{4}+2 \mu^{2} \nu^{2}+\nu^{4}\right)$, respectively.

Note that the expressions in Lemma 3 correspond to generalized LI cancellation schemes. With linear CB-N or ZF$\mathrm{N}$ precoding, under perfect $\mathrm{CSI}$, the $\mathrm{LI}$ in the UL can be suppressed. On the other hand, under linear $\mathrm{CB}$ or $\mathrm{ZF}$ precoders, the LI signals at the massive-antenna BS and at the MT are characterized in Lemma 3 with arbitrary Rician statistics, which can be tuned to capture the LI subtraction capability by design or through measurements [17]. 


$$
\begin{aligned}
& \mathcal{S}_{\mathrm{DL}}=\int_{0}^{+\infty} \mathbb{1}_{\mathrm{FD}}\left(\mathcal{M}_{\mathcal{L} \mathcal{I}_{\mathrm{DL}}}(z)\right) \mathbb{1}_{\mathrm{FD}}\left(\mathcal{M}_{\mathcal{C} \mathcal{I}_{\mathrm{DL}}}(z)\right) \int_{0}^{+\infty} \frac{\mathcal{M}_{\mathcal{I} \mathcal{C} \mathcal{I}_{\mathrm{DL} \mid r}}(z) \mathcal{M}_{\mathcal{M U} \mathcal{I}_{\mathrm{DL} \mid r}}(z)\left(1-\mathcal{M}_{\mathcal{X}_{\mathrm{DL}} \mid r}(z)\right)}{z \exp \left(z \sigma_{\mathrm{DL}}^{2}\right)} \mathcal{P}_{d_{\left[l_{0}, l_{k}\right]}}(r) \mathrm{d} z \mathrm{~d} r \\
& \mathcal{S}_{\mathrm{UL}}=\int_{0}^{+\infty} \mathbb{1}_{\mathrm{FD}}\left(\mathcal{M}_{\mathcal{L I}_{\mathrm{UL}}}(z)\right) \mathbb{1}_{\mathrm{FD}}\left(\mathcal{M}_{\mathcal{C I}_{\mathrm{UL}}}(z)\right) \int_{0}^{+\infty} \frac{\mathcal{M}_{\mathcal{I C I}_{\mathrm{UL} \mid r}}(z) \mathcal{M}_{\mathcal{M U \mathcal { U }} \mathcal{I}_{\mathrm{UL} \mid r}}(z)\left(1-\mathcal{M}_{\mathcal{X}_{\mathrm{UL}} \mid r}(z)\right)}{z \exp \left(z \sigma_{\mathrm{UL}}^{2}\right)} \mathcal{P}_{d_{\left[l_{0}, l_{k}\right]}}(r) \mathrm{d} z \mathrm{~d} r \\
& \chi_{\mathrm{I}}(z, p, r, \beta, u, v)=\frac{1}{\left(1+z p v r^{-\beta}\right)^{u}} \\
& \chi_{\mathrm{II}}(z, \lambda, p, \mathscr{E}, \beta, u, v)=\exp \left(-\pi \lambda\left[-\mathscr{E}^{2}+\frac{\mathscr{E}^{2}}{\left(1+z p v \mathscr{E}^{-\beta}\right)^{u}}+(z p v)^{\frac{2}{\beta}} \times\right.\right. \\
& \left.\left.\left(\frac{\Gamma\left(1-\frac{2}{\beta}\right) \Gamma\left(u+\frac{2}{\beta}\right)}{\Gamma(u)}-u \exp \left(-i \pi\left(u+\frac{2}{\beta}\right)\right) \mathcal{B}_{-\frac{\mathscr{S}^{\beta}}{z p v}}\left(u+\frac{2}{\beta},-u\right)\right)\right]\right)
\end{aligned}
$$

\begin{tabular}{|c|c|}
\hline $\mathcal{M}_{\mathcal{X}_{\mathrm{DL}} \mid r}(z)$ & $\chi_{\mathrm{I}}(z, p, r, \beta, u, v), p \rightarrow p_{\mathrm{BS}}, u \rightarrow \kappa\left(G_{\mathcal{X}_{\mathrm{DL}}}\right), v \rightarrow \theta\left(G_{\mathcal{X}_{\mathrm{DL}}}\right)$ \\
\hline $\mathcal{M}_{\mathcal{X}_{\mathrm{UL}} \mid r}(z)$ & $\chi_{\mathrm{I}}(z, p, r, \beta, u, v), p \rightarrow p_{\mathrm{MT}}, u \rightarrow \kappa\left(G_{\mathcal{X}_{\mathrm{UL}}}\right), v \rightarrow \theta\left(G_{\mathcal{X}_{\mathrm{UL}}}\right)$ \\
\hline $\mathcal{M}_{\mathcal{M U} \mathcal{I}_{\mathrm{DL} \mid r}}(z)$ & $\mathbb{1}_{(\mathbf{V} \neq \mathrm{ZF}, \mathrm{ZF}-\mathrm{N})}\left(\chi_{\mathrm{I}}(z, p, r, \beta, u, v)\right), p \rightarrow p_{\mathrm{BS}}, u \rightarrow \kappa\left(G_{\mathcal{M U} \mathcal{I}_{\mathrm{DL}}}\right), v \rightarrow \theta\left(G_{\mathcal{M U} \mathcal{I}_{\mathrm{DL}}}\right)$ \\
\hline $\mathcal{M}_{\mathcal{M U I} \mathcal{I}_{\mathrm{UL} \mid r}}(z)$ & $\mathbb{1}_{(\mathbf{W} \neq \mathrm{ZF})}\left(\chi_{\mathrm{I}}(z, p, r, \beta, u, v)\right), p \rightarrow p_{\mathrm{MT}}, u \rightarrow K \times \kappa\left(G_{\mathcal{M U} \mathcal{I}_{\mathrm{UL}}}\right), v \rightarrow \theta\left(G_{\mathcal{M U \mathcal { U }}}{ }_{\mathrm{UL}}\right)$ \\
\hline $\mathcal{M}_{\mathcal{I C} \mathcal{I}_{\mathrm{DL} \mid r}}(z)$ & $\chi_{\mathrm{II}}(z, \lambda, p, \mathscr{E}, \beta, u, v), \lambda \rightarrow \lambda_{\mathrm{BS}}, p \rightarrow p_{\mathrm{BS}}, \mathscr{E} \rightarrow r, u \rightarrow \kappa\left(G_{\mathcal{I C}_{\mathcal{I}}}\right), v \rightarrow \theta\left(G_{\mathcal{I C C}_{\mathrm{DL}}}\right)$ \\
\hline $\mathcal{M}_{\mathcal{I C} \mathcal{I}_{\mathrm{UL} \mid r}}(z)$ & $\chi_{\mathrm{II}}(z, \lambda, p, \mathscr{E}, \beta, u, v), \lambda \rightarrow K \times \lambda_{\mathrm{BS}}, p \rightarrow p_{\mathrm{MT}}, \mathscr{E} \rightarrow r, u \rightarrow \kappa\left(G_{\mathcal{I C I}_{\mathrm{UL}}}\right), v \rightarrow \theta\left(G_{\mathcal{I C I}_{\mathrm{UL}}}\right)$ \\
\hline $\mathcal{M}_{\mathcal{C} \mathcal{I}_{\mathrm{DL}}}(z)$ & $\chi_{\mathrm{II}}(z, \lambda, p, \mathscr{E}, \beta, u, v), \lambda \rightarrow K \times \lambda_{\mathrm{BS}}, p \rightarrow p_{\mathrm{MT}}, \mathscr{E} \rightarrow 0, u \rightarrow \kappa\left(G_{\mathcal{C} \mathcal{I}_{\mathrm{DL}}}\right), v \rightarrow \theta\left(G_{\mathcal{C} \mathcal{I}_{\mathrm{DL}}}\right)$ \\
\hline $\mathcal{M}_{\mathcal{C} \mathcal{I}_{\mathrm{UL}}}(z)$ & $\chi_{\mathrm{II}}(z, \lambda, p, \mathscr{E}, \beta, u, v), \lambda \rightarrow \lambda_{\mathrm{BS}}, p \rightarrow p_{\mathrm{BS}}, \mathscr{E} \rightarrow 0, u \rightarrow \kappa\left(G_{\mathcal{C} \mathcal{I}_{\mathrm{UL}}}\right), v \rightarrow \theta\left(\mathcal{C I}_{\mathrm{UL}}\right)$ \\
\hline $\mathcal{M}_{\mathcal{L} \mathcal{I}_{\mathrm{DL}}}(z)$ & $\chi_{\mathrm{I}}(z, p, r, \beta, u, v), p \rightarrow p_{\mathrm{MT}}, r \rightarrow 1, u \rightarrow \kappa\left(G_{\mathcal{L I}_{\mathrm{DL}}}\right), v \rightarrow \theta\left(G_{\mathcal{L} \mathcal{I}_{\mathrm{DL}}}\right)$ \\
\hline $\mathcal{M}_{\mathcal{L} \mathcal{I}_{\mathrm{UL}}}(z)$ & $\mathbb{1}_{(\mathbf{V} \neq \text { ZF-N,CB-N) }}\left(\chi_{\mathrm{I}}(z, p, r, \beta, u, v)\right), p \rightarrow p_{\mathrm{BS}}, r \rightarrow 1, u \rightarrow \kappa\left(G_{\mathcal{L I}_{\mathrm{UL}}}\right), v \rightarrow \theta\left(G_{\mathcal{L I}_{\mathrm{UL}}}\right)$ \\
\hline
\end{tabular}

TABLE III: Different useful and interfering signals MGFs in the multi-cell multi-user LSAS.

\section{ERgodic Rate Analysis}

In the following lemma, we provide analytical expressions for the computation of the LSAS ERs (in nat $/ \mathrm{s} / \mathrm{Hz}$ ) without requiring the need for the knowledge of the SINRs distributions (which are not available in general).

Lemma 3. The LSAS ERs in the DL, $\left.\mathcal{E}\left\{\log \left(1+\mathcal{Y}_{D L}\right)\right\}\right)$, and in the $U L, \mathcal{E}\left\{\log \left(1+\mathcal{Y}_{U L}\right)\right\}$, are respectively given in $\mathrm{nat} / \mathrm{s} / \mathrm{Hz}$ by (9) and (10).

Proof: The proof follows immediately from [18, Lemma 1] and is therefore omitted.

Due to the constraints imposed by the cellular association strategy and multiple MTs served per massive-antenna BS in the LSAS under study, there exists inherent dependencies in the active MTs set [19]. Here, for the sake of analytical tractability, we invoke the approach from [20] by approximating the UL interfering terms using spatially-thinned PPPs.

The MGFs of the different useful and interfering signals in the multi-cell multi-user LSAS are derived next.
Lemma 4. By defining the general functions (11) and (12), the MGFs of the LSAS useful and interfering signals are characterized in Table III.

Proof: The result follows from a similar approach to that in [21] and is hence omitted due to space limitations.

The results from Lemmas 1-4 allow for the efficient calculation of the multi-cell multi-user LSAS ERs in the DL and $\mathrm{UL}$ in both FD and HD modes of communications. In what follows, let $\mathcal{S}_{\mathrm{DL}}^{\mathrm{FD}}\left(\mathcal{S}_{\mathrm{DL}}^{\mathrm{HD}}\right)$ and $\mathcal{S}_{\mathrm{UL}}^{\mathrm{FD}}\left(\mathcal{S}_{\mathrm{UL}}^{\mathrm{HD}}\right)$ respectively denote the DL and UL ERs in the FD (HD) LSAS. In order to facilitate performance comparison, we consider the transmission/reception over two resource blocks. Hence, the corresponding FD versus HD gain can be expressed as

$$
\frac{\mathbb{S}_{\mathrm{FD}}}{\mathbb{S}_{\mathrm{HD}}}=\frac{2\left(\mathcal{S}_{D L}^{\mathrm{FD}}+\mathcal{S}_{U L}^{\mathrm{FD}}\right)}{\mathcal{S}_{D L}^{\mathrm{HD}}+S_{U L}^{\mathrm{HD}}} .
$$

The characterization of the system performance in this paper facilitates performance analysis and optimization under generalized settings of LSAS parameters. The exact FD versus HD 


$$
\mathbb{S}_{\mathrm{FD}}=\int_{0}^{+\infty} \frac{2}{\frac{s}{2}+\operatorname{arccot}(s)}\left(2-\left(1+\frac{1}{s^{2}}\right)^{-N}-\left(1+\frac{1}{s^{2}}\right)^{-M}\right) \mathrm{d} s
$$

$$
\mathbb{S}_{\mathrm{HD}}=\int_{0}^{+\infty} \frac{2}{s+\operatorname{arccot}(s)}\left(1-\left(1+\frac{1}{s^{2}}\right)^{-N}-\left(1+\frac{1}{s^{2}}\right)^{-M}\right) \mathrm{d} s
$$

LSAS ER gain, however, cannot be obtained in closed-form as a result of the ER expressions involving multi-fold improper integrals. We do however provide results in the following special case of interest.

Lemma 5. With complete subtraction of the LI, equivalent transmit power in the DL and UL, interference-limited region, and special case of path-loss exponent being equal to four, the $F D$ and HD ERs in the multi-cell single-user LSAS over two resource blocks are respectively given by (14) and (15).

Proof: The results are derived using substitution $z \rightarrow \frac{u^{4}}{p}$, Cartesian-polar conversion with $r \rightarrow l \sin (\psi), u \rightarrow l \cos (\psi)$, with the Jacobian $l$, and further utilizing the integral identity $\int_{0}^{+\infty} l \exp \left(-l^{\alpha}\right) \mathrm{d} l=\frac{1}{2} \Gamma\left(1+\frac{2}{\alpha}\right), \alpha>0$.

The simplified ER expressions derived above cannot be reduced further in terms of elementary functions. Hence, a closed-form expression for the FD versus HD ER gain cannot be obtained for this special case. We proceed by devising a tight bounded expression of the corresponding gain in the special case under consideration.

Lemma 6. Considering the special case in Lemma 5, with the antenna array size at the massive-antenna BSs being $\mathcal{A}$, the ER gain of FD versus HD LSAS can be upper-bounded as $\frac{\mathbb{S}_{F D}}{\mathbb{S}_{H D}}<2 \min \left(\frac{\log (3)}{2} \mathcal{A}^{\frac{1}{20}}, 1\right)$.

Proof: This result is obtained through non-linear curve fitting of the exact (large) data.

The result from Lemma 6 infers that the ER gain of FD over HD LSAS - even under complete LI subtraction - increases only logarithmically in the antenna array size. It is only in the asymptotic antenna region, $N \rightarrow \infty, M \rightarrow \infty$, that the exact $\frac{\mathbb{S}_{\mathrm{FD}}}{\mathbb{S}_{\mathrm{HD}}}$ converges to two.

\section{NumericAl EXAMPLES}

In this section, we utilize the proposed unified framework to evaluate the multi-cell multi-user LSAS ER performance in both FD and HD communications modes. At the same time, the validity of the theoretical findings is examined through Monte-Carlo (MC) simulations from running $100 \mathrm{k}$ trials inside a circular region of radius $100 \mathrm{~km}$. The fixed simulation parameters are: $\lambda_{\mathrm{BS}}=\frac{4}{\pi} \mathrm{km}^{-2}$ (massive-antenna BS density), $F_{c}=1 \mathrm{GHz}$ (carrier frequency), $B=10 \mathrm{MHz}$ (total bandwidth), and $\beta=4$ (path-loss exponent). Moreover, we incorporate the notion that, in LSAS with perfect CSI, the high antenna array gain allows the transmit power to be linearly conserved in the number of antennas [3]. Note that all results correspond to the sum ER over two resource blocks.

\section{A. Impact of Loop-Interference}

We first investigate the ER performance for a (practical) LSAS setup with 64 antennas (currently under investigation in 3GPP, Study Item $R P$-141831). In FD mode, we employ linear ZF-N precoding (for suppressing multi-user and loop-back interferences in the UL) and ZF decoding (for suppressing multi-user interference in the DL). In HD mode, on the other hand, linear ZF precoder and decoder are adopted at the transmit and receive sides, respectively. The ER gain of FD versus HD LSAS in the DL, UL, and total (DL + UL) are depicted with different LI subtraction levels at the MT side in Fig. 1. Intuitively, with improved LI subtraction capability at the MTs, the FD ER performance versus that in HD increases. On the other hand, it can be seen that even by managing to subtract LI below noise level, the improvement in performance may be viewed as subpar. For instance, approximately, with an LI channel power attenuation of $-120 \mathrm{~dB}$, a total ER gain of $\times 1.25$ from FD over HD operation can be achieved (resulting from $\times 1.65$ increase in the UL ER and $\times 0.92$ decrease in the DL ER, respectively). This trend results from (i) a loss of antenna array gain, i.e., $(M-N-K+1)-(M-K+1)=-N$, for spatially suppressing LI at the massive-antenna BS side in FD mode, and (ii) the effect of significantly lower interference level in HD mode (not experiencing LI and CI).

\section{B. Impact of Antenna Array Size}

Next, we study the effect of antenna array size on the FD versus HD total ER performance gain in interference-limited region in Fig. 2. Here, in FD mode, we consider two different cases, namely at the massive-antenna BSs, with (i) linear CB$\mathrm{N}$ precoding for LI suppression (blue curves), and (ii) linear CB precoding with perfect LI subtraction (red curves). The latter scheme corresponds to the case upper-bounded in closedform in Lemma 6 (black curve). Note that in the former scheme, the antenna array is divided in accordance to the optimal transmit to receive antenna ratio $\frac{N}{M} \approx \frac{1}{3}$ depicted in [8]. Specifically, for an antenna array size $\mathcal{A}$, we use $N=\max \left(\frac{1}{4}\right.$ Integer-Part $\left.[\mathcal{A}], 1\right), M=\mathcal{A}-N$. In addition, here, we also consider the case where the LI at the MT side is subtracted perfectly. In HD mode, we employ linear conjugatebeamforming. Note that the $\mathrm{x}$-axis in Fig. 2 is in base-two logarithmic scale. We can observe that the FD versus HD total ER gain increases only logarithmically in the antenna array size. In addition, the $\times 2$ ER improvement, when comparing FD with HD mode, is only achieved as the number of antennas goes to infinity under both LI spatial and subtraction schemes. 
FD versus HD LSAS

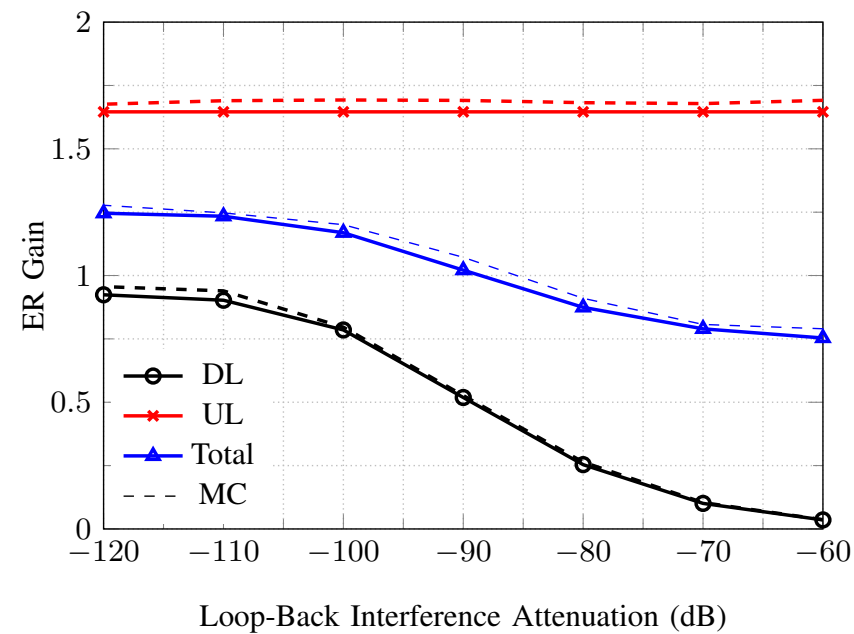

Fig. 1: Simulation parameters: $\lambda_{\mathrm{BS}}=\frac{4}{\pi} \mathrm{km}^{-2}, M=48, N=16$, $K=2, p_{\mathrm{BS}}=\frac{1}{M} \mathrm{~W}, p_{\mathrm{MT}}=\frac{1}{N} \mathrm{~W}, B=10 \mathrm{MHz}, \sigma_{\mathrm{DL}}^{2}=\sigma_{\mathrm{UL}}^{2}=-90$ $\mathrm{dBm}, \frac{\mu^{2}}{2 \nu^{2}}=1$ (Rician factor), $\beta=4$.

\section{SUMMARY}

In this work, we utilized tools from stochastic geometry theory to devise a tractable analytical framework for the design and analysis of multi-cell multi-user LSAS in both FD and HD modes of communications. The Rician distribution was used to model the LI channels in FD mode, and other channels were characterized using the Rayleigh distribution. The statistical distributions of the different useful and interfering signals were derived under linear $\mathrm{CB}$ and $\mathrm{ZF}$ processes, including a new tight Gamma approximation for the LI at the massive-antenna BSs. We provided analytical expressions for the computation of the LSAS ERs in the DL and UL, with MGFs of the different signals involved derived in closed-form. The results demonstrated that the anticipated $\times 2$ FD versus $H D$ gain in ER performance is only achieved in the asymptotic antenna region.

\section{REFERENCES}

[1] SoftBank Group Corp., "Massive MIMO," www.softbank.jp/mobile/ network/explanation/massive-mimo, 2016.

[2] E. G. Larsson, O. Edfors, F. Tufvesson, and T. L. Marzetta, "Massive MIMO for next generation wireless systems," IEEE Commun. Mag., vol. 52, no. 2, pp. 186-195, Feb. 2014.

[3] L. Lu, G. Y. Li, A. L. Swindlehurst, A. Ashikhmin, and R. Zhang, "An overview of massive MIMO: Benefits and challenges," IEEE J. Sel. Topics Signal Process., vol. 8, no. 5, pp. 742-758, Oct. 2014.

[4] M. Duarte, C. Dick, and A. Sabharwal, "Experiment-driven characterization of full-duplex wireless systems," IEEE Trans. Wireless Commun., vol. 11, no. 12, pp. 4296-4307, Dec. 2012.

[5] H. Alves, R. D. Souza, and M. E. Pellenz, "Brief survey on full-duplex relaying and its applications on 5G," in Proc. IEEE Int. Workshop Comput. Aided Modeling Anal. Design Commun. Links Netw. (CAMAD), Sept. 2015, pp. 17-21.

[6] D. Korpi, J. Tamminen, M. Turunen, T. Huusari, Y. S. Choi, L. Anttila, S. Talwar, and M. Valkama, "Full-duplex mobile device: pushing the limits," IEEE Commun. Mag., vol. 54, no. 9, pp. 80-87, Sep. 2016.

[7] T. Riihonen, M. Vehkaper, and R. Wichman, "Large-system analysis of rate regions in bidirectional full-duplex MIMO link: Suppression versus cancellation," in Proc. Inf. Sci. and Syst. Conf. (CISS), Mar. 2013, pp. $1-6$.
FD versus HD LSAS

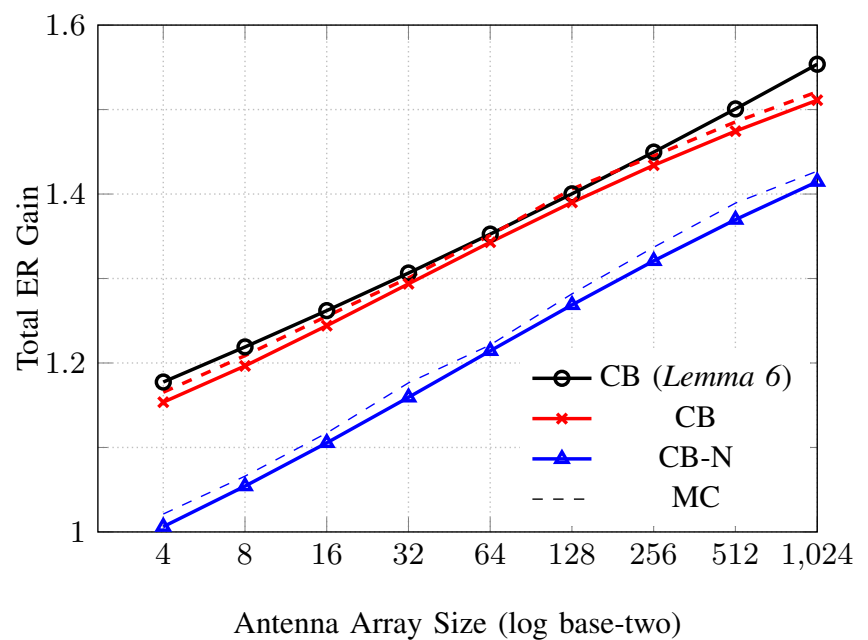

Fig. 2: Simulation parameters: $\lambda_{\mathrm{BS}}=\frac{4}{\pi} \mathrm{km}^{-2}, K=1, p_{\mathrm{BS}}=\frac{1}{M}$ $\mathrm{W}, p_{\mathrm{MT}}=\frac{1}{N} \mathrm{~W}, B=10 \mathrm{MHz}, \sigma_{\mathrm{DL}}^{2}=\sigma_{\mathrm{UL}}^{2}=0, \beta=4$.

[8] B. Yin, M. Wu, C. Studer, J. R. Cavallaro, and J. Lilleberg, "Full-duplex in large-scale wireless systems," in Proc. Asilomar Conf. Signals, Syst. and Comput. (ASILOMAR), Nov. 2013.

[9] J. Bai and A. Sabharwal, "Large antenna analysis of multi-cell fullduplex networks," arXiv:1606.05025, 2016.

[10] C. Psomas, M. Mohammadi, I. Krikidis, and H. A. Suraweera, "Directional antennas for interference management in full-duplex cellular networks," arXiv:1602.02718, 2016.

[11] M. Di Renzo, W. Lu, and P. Guan, "The intensity matching approach: A tractable stochastic geometry approximation to system-level analysis of cellular networks," arXiv:1604.02683, 2016.

[12] A. Shojaeifard, K.-K. Wong, M. Di Renzo, G. Zheng, K. A. Hamdi, and J. Tang, "Massive MIMO-enabled full-duplex cellular networks," arXiv:1611.03854, 2016.

[13] A. Shojaeifard, K. A. Hamdi, E. Alsusa, D. K. C. So, and J. Tang, "Exact SINR statistics in the presence of heterogeneous interferers," IEEE Trans. Inf. Theory, vol. 61, no. 12, pp. 6759-6773, Dec. 2015.

[14] E. Björnson, E. G. Larsson, and T. L. Marzetta, "Massive MIMO: ten myths and one critical question," IEEE Commun. Mag., vol. 54, no. 2, pp. 114-123, Feb. 2016.

[15] A. AlAmmouri, H. ElSawy, O. Amin, and M. S. Alouini, "In-band $\alpha$ duplex scheme for cellular networks: A stochastic geometry approach," IEEE Trans. Wireless Commun., vol. 15, no. 10, pp. 6797-6812, Oct. 2016.

[16] M. Haenggi, J. G. Andrews, F. Baccelli, O. Dousse, and M. Franceschetti, "Stochastic geometry and random graphs for the analysis and design of wireless networks," IEEE J. Sel. Areas Commun., vol. 27, no. 7, pp. 1029-1046, 2009.

[17] I. Atzeni and M. Kountouris, "Full-duplex MIMO small-cell networks: Performance analysis," in Proc. IEEE Global Commun. Conf. (GLOBECOM), Dec. 2015, pp. 1-6.

[18] K. A. Hamdi, "A useful lemma for capacity analysis of fading interference channels," IEEE Trans. Commun., vol. 58, no. 2, pp. 411-416, Feb. 2010.

[19] T. D. Novlan, H. S. Dhillon, and J. G. Andrews, "Analytical modeling of uplink cellular networks," IEEE Trans. Wireless Commun., vol. 12, no. 6, pp. 2669-2679, June 2013.

[20] M. D. Renzo and P. Guan, "Stochastic geometry modeling and systemlevel analysis of uplink heterogeneous cellular networks with multiantenna base stations," IEEE Trans. Commun., vol. 64, no. 6, pp. 24532476, June 2016.

[21] A. Shojaeifard, K. A. Hamdi, E. Alsusa, D. K. C. So, J. Tang, and K. K. Wong, "Design, modeling, and performance analysis of multi-antenna heterogeneous cellular networks," IEEE Trans. Commun., vol. 64, no. 7, pp. 3104-3118, July 2016. 